\title{
Static analysis of a torsion motor generating flexion - extension motions of the elbow
}

\author{
Tudor Deaconescu* and Andrea Deaconescu \\ Transilvania University of Braşov, Department of Industrial Engineering and Management, Bd. \\ Eroilor 29, Braşov, Romania
}

\begin{abstract}
The paper presents the construction and static analysis of a rotation module responsible for conducting flexion-extension motions as part of a medical rehabilitation device of the elbow. The rotation module is actuated by a torsion motor consisting of a pair of agonist-antagonist pneumatic muscles. The study concerning the static behaviour of the rotation module draws upon similarity to the biological model, considering the influence of the neuronal control quantities on the forces developed by the two muscles.
\end{abstract}

\section{Introduction}

The elbow is one of the most sensitive joints of the human body, subjected to considerable strain due to its visible location between the shoulder joint and the wrist. The functional importance of the elbow is proved by the impact of injury inflicted on it, often resulting in the impossibility of conducting even the most basic activities of the upper limb.

Rigidity or diminished mobility of the elbow consequently to trauma and surgical interventions is one of the most common problems encountered in medical practice. It has been clinically proven that addressing the issued by prolonged immobilisation has negative effects on the human body, extending the period necessary for recovery and slowing down the healing process. In order to prevent such undesired evolution, the affected joint should be mobilised as soon as possible, not later than 2-3 days after the occurrence of the trauma [1]. While natural recovery of mobility can take as long as several months, applying adequate recovery methods prevents the forming of scar tissue, diminished joint rigidity and accelerates healing.

Physical therapy being a motion-based technique is the most important method used for recovering the functional balance of the body, in order to restore diminished functions and increase joint mobility. One of the most efficient techniques deployed by physical therapy is the dynamic approach, based on continuous passive motion.

Passive continuous motions can be performed by a physical therapist or by specially conceived equipment. Devices for continuous passive motion ensure enhanced comfort and safe work with the patient, what recommends them for post-traumatic recovery. Such equipment was developed in order to replace the physical work of the therapist with similar and even superior results.

\footnotetext{
${ }^{*}$ Corresponding author: tdeacon@unitbv.ro
} 
In paper [2] the authors discuss a study conducted on 81 patients who had undergone surgery for restoring the mobility of the elbow ligaments and who subsequently have benefitted from rehabilitation by CPM (Continuous Passive Motion). The results revealed that the average amplitude of motion had improved from $69^{\circ}$ to $109^{\circ}$; further the function of the upper limb was improved and pain reduced from 32 to 16 and from 20 to 10 according to the assessment made by means of DASH scores.

Paper [3] presents a comparison between the rehabilitation of the elbow performed at the medical facility and at the patient's home, respectively, by means of continuous passive motion (CPM) equipment. It could be concluded that a short period of hospitalisation, of 24 hours, followed by two weeks of CPM-based therapy at the patient's home is the equivalent of 72 hours of hospitalisation.

Further on the paper presents and discusses the construction and static analysis of a rotation module responsible for conducting flexion-extension motions as part of a medical rehabilitation device of the elbow. The rotation module is actuated by a torsion motor consisting of a pair of agonist-antagonist pneumatic muscles.

\section{Structure and kinematics of the elbow rehabilitation equipment}

The functional analysis of the elbow joint capsule reveals two different kinematic rotational pairs: one ensuring the movements of flexion and extension, and another one for pronation and supination. Studies conducted on the biomechanics of the elbow joint have yielded the variation limits of the angles required for flexion-extension and pronation-supination, respectively. These are of $-90^{\circ} \ldots+50^{\circ}$ (amplitude of $140^{\circ}$ ) for flexion-extension, and of $\pm 90^{\circ}$ for pronation-supination, respectively.

Figure 1 presents the kinematic diagram of the biomechanical model of the elbow, in this case a mechanical kinematic pair with two degrees of freedom, with the axes of rotation forming a $90^{\circ}$ angle.

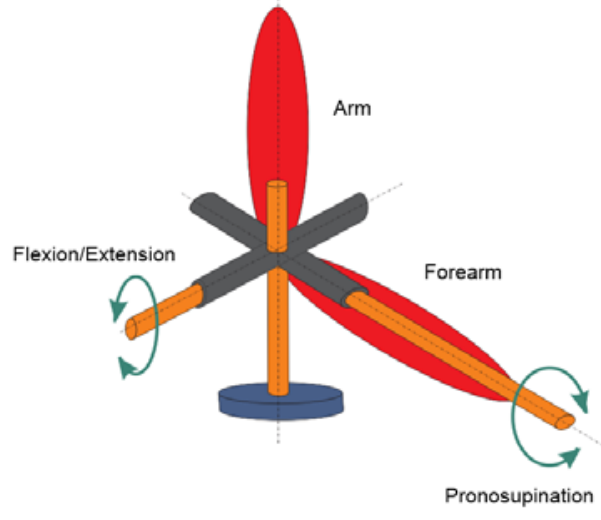

Fig. 1. Kinematic diagram of the biomechanical model of the elbow joint.

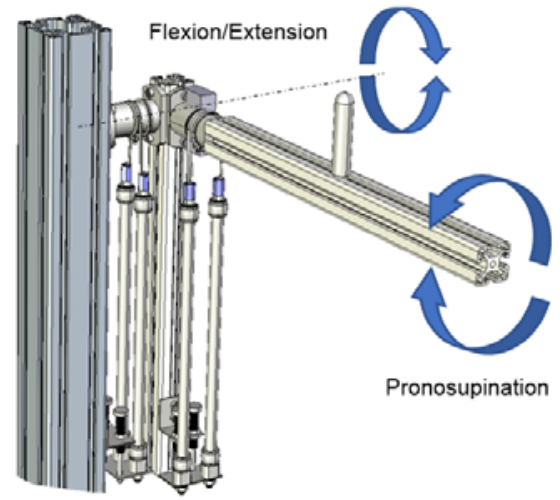

Fig. 2. Construction of the elbow rehabilitation joint.

Figure 2 presents the construction of the elbow rehabilitation equipment, each of the two rotation motions being generated by a torsion motor consisting of a pair of agonist-antagonist pneumatic muscles.

Further on presented is the construction and static analysis of the rotation module responsible for flexion-extension. In order to generate this motion a rotational module is deployed actuated by a pair of pneumatic muscles, which operating in agonist-antagonist mode generate a rotation of an amplitude greater than $140^{\circ}$ and ensure the equilibrium of a certain intermediary position of the actuated system. 
The generation of rotation by pneumatic muscles is similar to a high degree to the same motion generated by human agonist-antagonist muscles, as shown in Figure 3. It can be noticed that contracting/relaxing the biceps and triceps in against-antagonist mode the forearm performs the movements of flexion and extension.
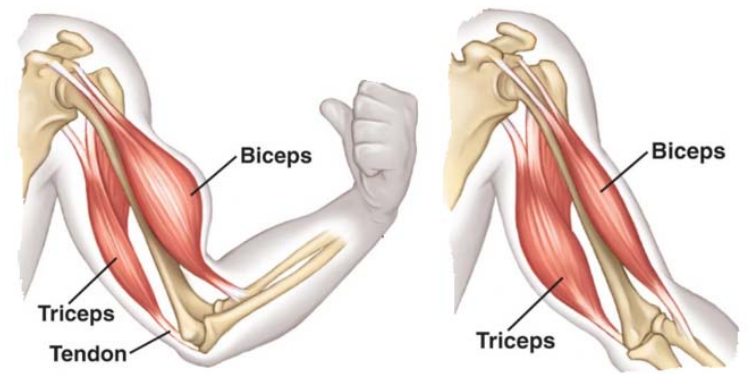

Fig. 3. Agonist-antagonist operation of the biceps and triceps.

\section{Static analysis of the rotation module responsible for flexion - extension}

As in the case of the biceps and the triceps, in the case of a mimetic mechanical system generating rotation two motors working have to be linked in agonist-antagonist mode. While one of these rotates a load, the other one acts like a brake such as to stop the motion in a certain position of balance. When the direction of motion changes, the roles of the two motors are inverted. Figure 4 presents the working principle of the two muscles denoted by M1 and M2. In the presented case the conducted movement is flexion.
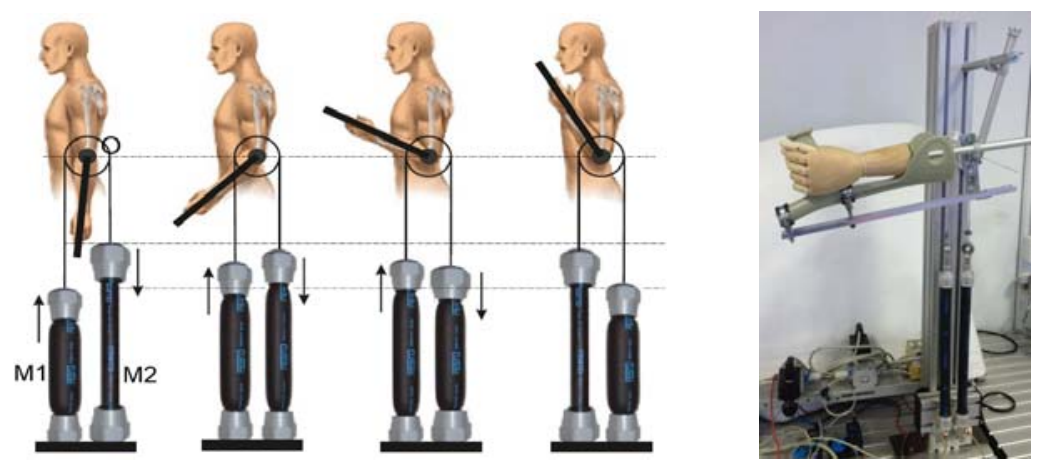

Fig. 4. Working principle and construction of the torsion motor.

Two flexible steel cables are connected to the upper ends of the pneumatic muscles. These are drawn over o pulley and fixed onto it, the winding angle of each cable over the pulley being of $270^{\circ}$. This constructive schematic allows the two muscles to cause, by inflating and deflating alternatingly, to cause the rotation of the pulley in one or the other direction.

The construction of the torsion motor includes two DMSP-20-300N-RM-CR pneumatic muscles manufactured by Festo, Germany, of $20 \mathrm{~mm}$ diameter and $300 \mathrm{~mm}$ length of the active part [4]. The axial contraction of these muscles when fed compressed air at a pressure of 6 bar is of $\Delta L_{\max }=60 \mathrm{~mm}$ ( $20 \%$ of the initial length).

The study concerning the static behaviour of the torsion module draws upon similarity to the biological model, considering the influence of the neuronal control quantities on the forces developed by the two muscles. In this case the general equation that describes the evolution of the static force developed by one muscle is: 


$$
F=u \cdot F_{\max } \cdot\left(1-\frac{\varepsilon}{\varepsilon_{\max }}\right)
$$

where $F_{\max }$ denotes the maximum force produced by the muscles when the specific axial contraction $\varepsilon$ is zero, and $u$ is a neuronal control quantity its values ranging from 0 to $1(0 \leq$ $u \leq 1$ ) [5]. The nerve impulses (the control quantities $u$ ) are generated by the alpha motoneurons, causing the muscle fibres to contract and implicitly generating force and displacement.

Under the antagonist action of the two muscles, a torque is generated in the joint described by equation 2 :

$$
T=R \cdot\left(F_{1}-F_{2}\right)
$$

where $F_{1}$ and $F_{2}$ are the forces developed by the two muscles (the agonist and the antagonist one), and $R$ - is the radius of the pulley that materializes the elbow joint $(R=19.1 \mathrm{~mm})$.

Applying equation (1) to each of the resulting forces yields relationships (3) and (4):

$$
\begin{aligned}
& F_{1}=u_{1} \cdot F_{\text {max }} \cdot\left(1-\frac{\varepsilon_{1}}{\varepsilon_{\text {max }}}\right) \\
& F_{2}=u_{2} \cdot F_{\text {max }} \cdot\left(1-\frac{\varepsilon_{2}}{\varepsilon_{\text {max }}}\right)
\end{aligned}
$$

where the control quantities $u$ satisfy the condition: $\mathrm{u}_{1}+\mathrm{u}_{2}=1$.

For a rotation of the joint to the maximum value $\theta_{\max }$, the specific axial contractions of the two muscles become:

$$
\begin{array}{ll}
\varepsilon_{1}=\varepsilon_{0}+\frac{R \cdot \theta_{\max }}{L_{i}}=\frac{\Delta L_{\max }}{L_{i}} \cdot 100 & {[\%]} \\
\varepsilon_{2}=\varepsilon_{0}-\frac{R \cdot \theta_{\max }}{L_{i}}=0 & {[\%]}
\end{array}
$$

where $\varepsilon_{0}$ denotes the specific axial contraction of the pneumatic muscle in the preloading phase $\left(\varepsilon_{0}=\Delta L_{\max } /\left(2 \cdot L_{i}\right)\right)$, and $L_{i}$ is the length of the muscle in its initial, relaxed state.

Considering the equations above the torque in the elbow joint becomes:

$$
T=R \cdot F_{\max } \cdot\left(1-\frac{\varepsilon_{0}}{\varepsilon_{\max }}\right) \cdot\left(u_{1}-u_{2}\right)-\frac{F_{\max } \cdot R^{2}}{L_{i} \cdot \varepsilon_{\max }} \cdot\left(u_{1}+u_{2}\right) \cdot \theta
$$

Upon introducing the notations:

$$
\begin{gathered}
K_{1}=R \cdot F_{\max } \cdot\left(1-\frac{\varepsilon_{0}}{\varepsilon_{\max }}\right) \\
K_{2}=\frac{F_{\max } \cdot R^{2}}{L_{i} \cdot \varepsilon_{\max }}
\end{gathered}
$$

equation (7) can be written as:

$$
T=K_{1} \cdot\left(u_{1}-u_{2}\right)-K_{2} \cdot\left(u_{1}+u_{2}\right) \cdot \theta
$$

For the concrete analysed case where two DMSP-20-300N-RM-CR pneumatic muscles are used, based on their technical specifications and the information provided by MuscleSim v. 2.0.1.5 programme, the data of interest necessary for performing the calculations are: $\varepsilon_{\max }$ $=20 \% ; \Delta L_{\max }=60 \mathrm{~mm} ; \varepsilon_{0}=10 \% ; F_{\max }=1552,9 \mathrm{~N}[6]$. With these values the maximum rotation angle to one or to the other side of the position of equilibrium is $\theta_{\max }= \pm \pi / 2$.

From equations (8) and (9) follows: 


$$
\begin{gathered}
K_{1}=R \cdot F_{\text {max }} \cdot\left(1-\frac{\varepsilon_{0}}{\varepsilon_{\max }}\right)=0,0191 \cdot 1552,9 \cdot\left(1-\frac{0.1}{0.2}\right)=14,83 \\
K_{2}=\frac{F_{\max } \cdot R^{2}}{L_{i} \cdot \varepsilon_{\max }}=\frac{1552,9 \cdot 0,0191^{2}}{0,3 \cdot 0,2}=9,44
\end{gathered}
$$

The maximum values of the torque in the joint are obtained when the two control quantities are $u_{1}=1$ and $u_{2}=0$ or $u_{1}=0$ and $u_{2}=1$, which situations correspond to a contraction that is maximum for one muscle and minimum for the other, respectively. In this case the maximum torque is:

$$
T_{\max }(\theta)=K_{1} \cdot(1-0)-K_{2} \cdot(1+0) \cdot \theta=K_{1}-K_{2} \cdot \theta
$$

Further on different values of the torques $T_{\max }(\theta)$ are calculated:

$$
\begin{gathered}
T_{\max }\left(-\frac{\pi}{2}\right)=K_{1}-K_{2} \cdot\left(-\frac{\pi}{2}\right)=14,83+9,44 \cdot \frac{\pi}{2}=29,65 \quad \mathrm{Nm} \\
T_{\max }(0)=K_{1}-K_{2} \cdot 0=14,83 \quad \mathrm{Nm} \\
T_{\max }\left(\frac{\pi}{2}\right)=K_{1}-K_{2} \cdot \frac{\pi}{2}=14,83-9,44 \cdot \frac{\pi}{2}=0,009 \quad \mathrm{Nm}
\end{gathered}
$$

Figure 5 presents a variation graph of the torque generated by the two muscles versus the rotation angle.

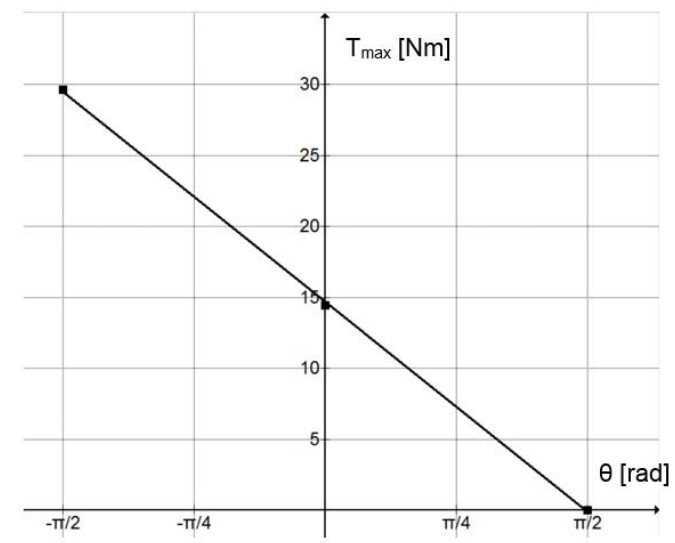

Fig. 5. Variation of the maximum torque versus the rotation angle.

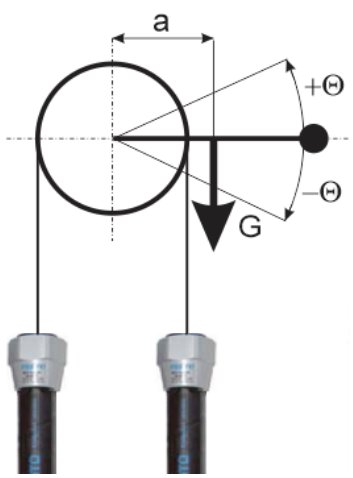

Fig. 6. Effect of the weight of the masses in motion on the rotation module.

Of interest for this study is also performing the so-called "gravity test" that considers the weight of the masses in motion. Figure 6 presents the schematic used for calculations with the following concrete data: $G$ - the sum of weights of the hand, forearm and hand support $(\mathrm{G}=0.46+1.2+3) \cdot 9.81=45.71 \mathrm{~N}) ; a$ - the distance to the axis of rotation of the application point of the weight $(\mathrm{a}=0.185 \mathrm{~m})$. With these data the torque generated by the weight is:

$$
T_{g}=(45.71 \cdot 0.185) \cdot \cos \theta=8.45 \cdot \cos \theta \quad[N \cdot m]
$$

Figure 7 presents the variation of the torque generated by the two muscles, of the torque caused by the masses in motion and of the total torque versus the rotation angle. 


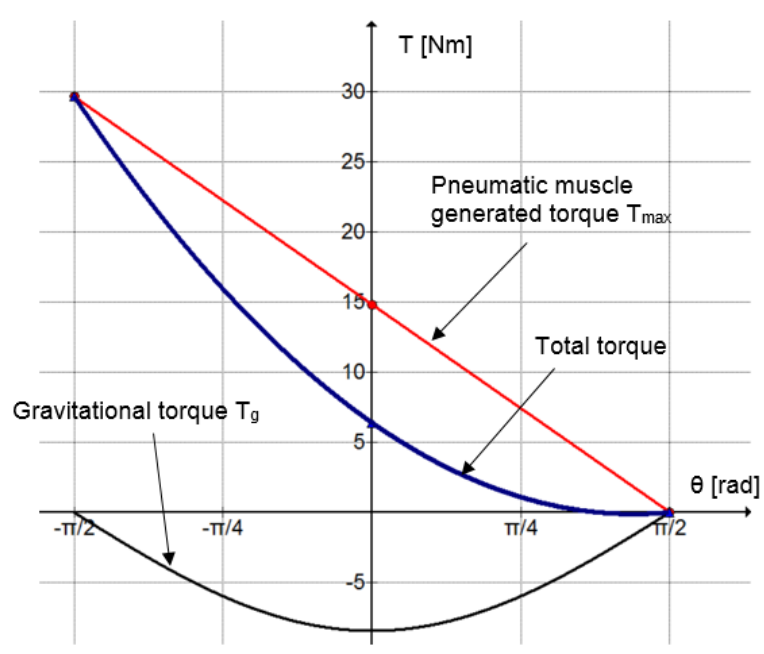

Fig. 7. Variation of the total torque in the joint of the rotation module.

\section{Conclusions}

This paper presents and discusses the construction of a torsion motor included in the structure of elbow rehabilitation equipment, being responsible for generating flexion-extension. The selected actuation solution involving two pneumatic muscles working antagonistically is a novel one, which to date been not deployed in rehabilitation equipment. The influence of the neuronal control quantities on the forces developed by the two muscles was taken into consideration for the completed static analysis. The calculations demonstrate that the total torque generated in the joint over its entire angular amplitude is sufficient for performing the rehabilitation exercises.

\section{References}

1. A. Deaconescu, T. Deaconescu. International MultiConference of Engineers and Computer Scientists Hong Kong II, 1872-1875 (2009)

2. Z.C.J. Higgs, B.A. Danks, M. Sibinski, L.A. Rymaszewski. The Bone and Joint Journal 94(3), 348-352 (2012)

3. H. Cruz et al. The Journal of The American Society of Anesthesiologists 123, 1256-1266 (2015)

4. Festo, Fluidic Muscle DMSP/MAS, https://www.festo.com/rep/en_corp/assets/pdf/info_501_en.pdf Accessed: 12.12.2016

5. N. Hogan. IEEE Transactions on Automatic Control AC-29(8), 681-690 (1984)

6. Festo MuscleSim v. 2.0.1.5., Festo MAS Fluidic Muscle Simulation Software (2005) 